\title{
Apoptosis in cervical squamous carcinoma: predictive value for survival following radiotherapy
}

\author{
J R Paxton, B S Bolger, A Armour, R P Symonds, J H Mao, R A Burnett
}

Department of Pathology, Western Infirmary, Glasgow G11 6NT, UK

J R Paxton

R A Burnett

\section{Department of}

Pathology, Royal

Victoria Infirmary,

Queen Victoria Road,

Newcastle upon Tyne

NE1 4LP, UK

B S Bolger

Beatson Oncology

Centre, Western

Infirmary, Glasgow

G11 6NT, UK

A Armour

R P Symonds

J H Mao

Correspondence to:

Dr Paxton

Accepted for publication 24 August 1999

\begin{abstract}
Background-Apoptosis, or programmed cell death, can be induced by radiotherapy. The extent of apoptosis in a tumour before treatment may have important implications for response to radiotherapy and long term survival.

Aim-To examine the extent of apoptosis in tumour tissue from patients with squamous carcinoma of the cervix before radiotherapy, and to correlate this with response to treatment and prognosis.

Methods-The percentage of apoptotic cells was assessed in 146 carcinomas of the cervix from patients scheduled to receive radiotherapy. The CAS 200 static image analysis system was used to count the number of tumour nuclei per high power field, while the numbers of apoptotic cells in the same field were visualised simultaneously on the image analyser and recorded manually.

Results-The median apoptotic level was $0.73 \%$. Patients were divided into two groups around the median. There was no statistically significant difference in outcome between the two groups as determined by long term survival following radiotherapy.

Conclusions-The CAS 200 static image analyser system can be used to assist in the rapid semiautomated assessment of apoptosis in conventionally prepared tissue. The results suggest that the apoptotic state of a tumour before treatment is of no value in predicting response to radiotherapy and subsequent prognosis. Tumour stage, size, and BrdU labelling index, as a measure of proliferation rate, remain the most important prognostic factors in terms of predicting local tumour control.

(F Clin Pathol 2000;53:197-200)
\end{abstract}

Keywords: apoptosis; uterine cervix; squamous cell carcinoma

Most anticancer treatment methods produce apoptosis in sensitive cells..$^{1-3}$ The tendency of a cancer cell to undergo apoptosis may have important implications for tumour progression and response to treatment. ${ }^{4}$ There is some evidence of a correlation between the extent of apoptosis in a tumour before treatment and its radiosensitivity. ${ }^{35}$

Given the potential implications of apoptosis on tumour kinetics it is not surprising that assessment of apoptosis in a range of neoplasms has aroused considerable interest. However, one of the principal problems that has hindered the investigation of apoptosis in tumours has been the lack of a satisfactory marker. This has made the quantitative assessment of apoptosis a laborious and time consuming exercise.

Various different techniques, including in situ end strand labelling of DNA fragments, ${ }^{67}$ immunocytochemical identification of associated genes and oncogenes, ${ }^{8}$ flow cytometric detection of cell shrinkage, ${ }^{9}$ diminished DNA binding of fluorescent dyes, ${ }^{10}$ and a range of special stain ${ }^{11}$ have been used to assist in the recognition of apoptosis but none of these is specific, and electron microscopy remains the gold standard. ${ }^{12}$

Light microscopic examination of haematoxylin and eosin (H\&E) stained sections remains one of the simplest and most reproducible methods. The morphology of apoptosis has been reviewed in detail by Wyllie. ${ }^{13}$ Furthermore, it is readily applicable to routinely processed material and is inexpensive and easy to interpret. In this study we attempt to circumvent some of the problems associated with the quantitative assessment of apoptosis in H\&E sections by using the CAS 200 image analysis system to count the background number of tumour cells in each field automatically while assessing the relatively small number of apoptotic cells per field manually.

\section{Methods}

PATIENT SELECTION

Over the two year study period (1991 to 1992) all patients with cervical carcinoma scheduled to receive radiotherapy at the Beatson Oncology Centre were approached to give written consent for entry into the study. Tumour tissue was collected at the initial staging procedure where tumour size and stage were recorded.

\section{TISSUE COLLECTION AND PROCESSING}

Multiple tumour samples were collected by obtaining additional punch biopsies at the time of the staging procedure choosing macroscopically viable areas of tumour. All tissue samples intended for microscopic assessment were subsequently fixed in formalin and routinely processed to paraffin wax. Sections $4 \mu \mathrm{m}$ thick were mounted on APES coated glass slides and stained with $\mathrm{H} \& \mathrm{E}$.

\section{ASSESSMENT OF APOPTOSIS}

Sufficient well preserved non-necrotic, formalin fixed, paraffin embedded tissue was present in 69 cases from the cohort available for analysis. All the cases included in this study were squamous carcinomas. For each of these cases, the percentage of apoptotic cells was assessed 
(by JRP) in representative $4 \mu \mathrm{m} \mathrm{H} \& \mathrm{E}$ stained sections. The CAS 200 static image analysis system (SIAS) was employed (Cell Analysis Systems Inc, Lombard, Illinois, USA). Images of all the nuclei of all the cells in the field are captured by a video camera using a $650 \mathrm{~nm}$ filter. Simple thresholding methods were used to set the lower limit for detection of nuclei, and this could be compared in real time with the image seen down the microscope. All sections were assessed at $\times 400$ magnification. Using this set up, individual tumour nuclei in a given high power field $(\times 400)$ could be readily identified and counted by the system in an automated fashion. The number of apoptotic cells per high power field (hpf) was visualised simultaneously both down the attached microscope and on the image analyser. The number of apoptotic cells per hpf $(\times 400)$ was generally small (range $0-8)$ and was recorded manually. Apoptotic cells were identified using widely accepted morphological criteria for apoptosis in $\mathrm{H} \& \mathrm{E}$ stained sections. ${ }^{13}$ All fields assessed were at least $1 \mathrm{hpf}$ away from areas of coagulative necrosis.

The image segmentation function on the program allowed non-neoplastic tissue to be excluded from the count. The image segmentation function on the CAS 200 SIAS was relatively easy to use. It involved drawing outlines with the mouse around the parts of the field that are to be included in the analysis. The segmented image was displayed on the computer monitor and this could be compared with the actual view of the field down the microscope. In most cases squamous carcinoma was present in confluent sheets, which could be readily delineated with the image segmentation function. In the small number of cases where the tumour was poorly differentiated with a more widely infiltrative pattern the image segmentation function could be used to draw round and isolate individual cells and small

Survival of patients split by size

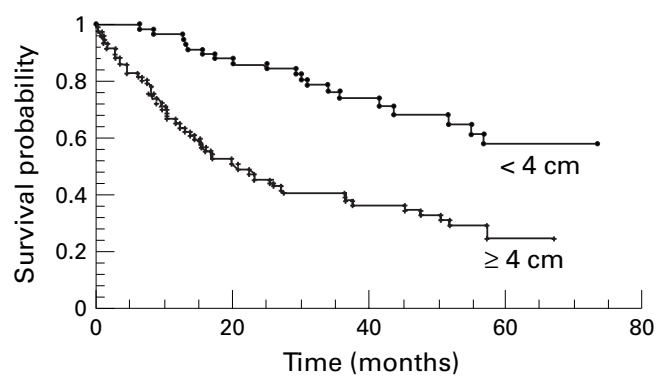

Survival of patients split by stage

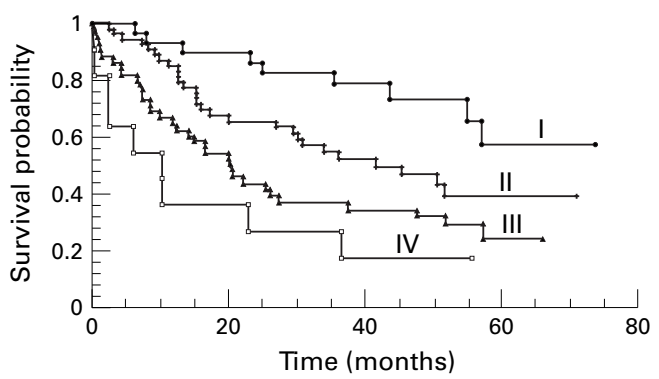

groups of cells, although this was more time consuming. Overlapping nuclei were not a problem as the abundant cytoplasm of squamous carcinoma cells tended to separate even poorly differentiated tumour cell nuclei.

In each case a minimum of 1000 tumour cells (approximately $10 \mathrm{hpf}$ ) were assessed and the total number of apoptotic cells recorded as a percentage of the total number of tumour cells.

\section{RADIOTHERAPY SCHEDULES}

For all the patients in this group, radiotherapy was the definitive mode of management. None underwent surgery to debulk their tumours.

Early lesions (stage I and II, $<5 \mathrm{~cm}$ ) received two intracavity insertions using the selectron after loading machine. The average A point dose was 36 Gy (from both insertions) at a dose rate of $1.8 \mathrm{~Gy} / \mathrm{h}$. This was followed by three weeks (four fractions) of treatment with 4 Met $x$ rays to parallel opposed diamond shaped fields, with the selectron treated area covered by a compensation wedge. The total summated pelvic side wall dose was 42.3 Gy given in 14 fractions with an A point dose of $61 \mathrm{~Gy} \pm 5 \%$.

More advanced lesions (bulky IIB, III, and IV) were treated over four weeks with external beam radiotherapy of $43 \mathrm{~Gy}$ in 20 fractions. This was followed by a selectron insertion of 26 Gy at $1.8 \mathrm{~Gy} / \mathrm{h}$.

No palliative doses were given.

FOLLOW UP

The patients were followed up on a regular basis with the most recent clinical update in December 1997. At this time 73 of the 146 patients were dead. Four had metastatic disease, four had recurrent local disease, one had both metastases and recurrent pelvic disease, and 64 had neither locally recurrent disease nor metastasis

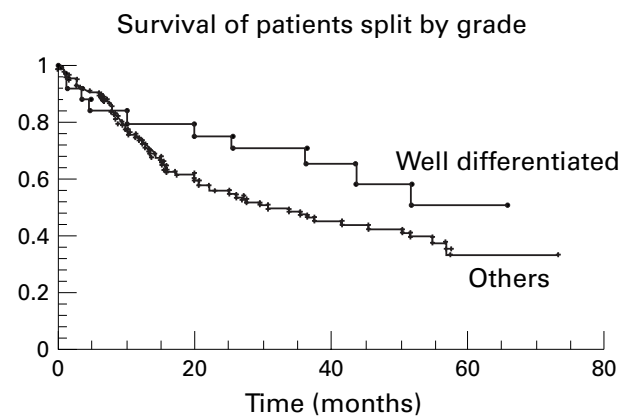

Survival of patients split by apoptosis

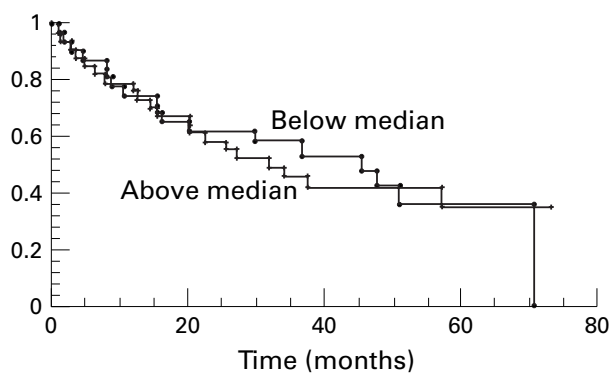

Figure 1 Comparison of survival of patients split by according to size, grade, stage, and apoptosis. 
Table 1 Patients divided according to stage of disease

\begin{tabular}{ll}
\hline Stage & $n$ \\
\hline I & 30 \\
II & 53 \\
III & 50 \\
IV & 13 \\
\hline
\end{tabular}

STATISTICAL ANALYSIS

Patients were divided into two groups around the median apoptotic count (expressed as a percentage of viable tumour cells). Both pelvic disease-free survival and overall survival for the two groups were compared using the negative log rank test and the results expressed in the form of Kaplan-Meier survival curves.

\section{Results}

The stage distribution of the 146 women in the study who had carcinoma of the cervix is outlined in table 1 .

The percentage of tumour cells undergoing spontaneous apoptosis before commencement of radiotherapy was determined 69 cases, all squamous carcinomas. The mean level of spontaneous apoptosis was $0.88 \%$. The range was $0.045-1.840 \%$ with a median of $0.73 \%$ and interquartile of $0.55-1.03 \%$.

STATISTICAL ANALYSIS OF SURVIVAL AND APOPTOSIS

Patients were divided into two groups depending on whether the extent of tumour apoptosis was above or below the median $(0.73 \%)$. There was no statistically significant difference in outcome as determined by pelvic disease-free survival or overall survival following radiotherapy, and the Kaplan-Meier survival curves for the two groups were almost identical (fig 1). A multivariate Cox analysis was done taking all the factors together to determine which were of independent significance. For disease-free survival tumour size and grade were significant with $p$ values of $<0.0001$ and 0.026 respectively. For overall survival tumour size was significant with a $\mathrm{p}$ value of $<0.0001$.

The follow up period for surviving patients ranged from 13 to 70 months, with a median of 51 months.

\section{Discussion}

This study has shown that it is possible to assess apoptosis in a semiautomated manner using the CAS 200 static image analysis system. The use of this system to count the background number of tumour nuclei made the assessment of larger amounts of tumour and larger numbers of cases considerably easier. The number of apoptotic cells visualised simultaneously on the image analyser was usually $\leqslant 4$ per hpf, and this could be readily counted and manually recorded. The image segmentation function on the program allowed non-neoplastic tissue to be reliably excluded from the count.

Radiotherapy and other treatments can induce apoptosis in sensitive cells ${ }^{1-3}$ and it has been suggested that there is a correlation between susceptibility to apoptosis and response to radiotherapy. ${ }^{3-5}$ The mechanism whereby radiotherapy triggers the apoptotic cascade in neoplastic cells is not completely understood but there is increasing evidence of altered function of the p53 tumour suppressor gene. ${ }^{414}{ }^{15}$ In this study we assessed spontaneous levels of apoptosis in squamous carcinoma of the cervix before the patients received radiotherapy. Our results suggest that the spontane- ous level of apoptosis in squamous carcinoma of the cervix is of no value in predicting overall or disease-free survival following radiotherapy. In a previous study on this cohort of patients it was shown that BrdU labelling index was significantly related to overall and disease-free survival ( $p=0.015) .{ }^{16}$ Tumour stage, size, and BrdU labelling index remain the most important prognostic factors in terms of predicting local tumour control (fig 1). In most cases the levels of spontaneous apoptosis were relatively small and maintained a constant narrow range; this is consistent with previous observations on certain other tumour types. ${ }^{17}$ Radiotherapy and other cancer treatments can induce apoptosis in sensitive tumour cells. ${ }^{1-3}$ We have not examined radiotherapy induced apoptosis. The mechanisms of spontaneous and induced apoptosis may be different. The capacity of radiotherapy to induce apoptosis in a tumour may not be related to the spontaneous or baseline level of apoptosis. A comparison of apoptosis levels in follow up biopsies after radiotherapy with the original baseline level of apoptosis would make an interesting future study and could be of more value in predicting response to radiotherapy and subsequent patient survival.

For most tumours it remains to be determined whether the degree of apoptosis correlates with other factors predicting biological behaviour. A positive correlation between the amount of apoptosis and other known prognostic factors has been reported for non-Hodgkin's lymphoma, ${ }^{18}$ prostatic adenocarcinoma, ${ }^{19}$ adrenal cortical tumours, ${ }^{20}$ and carcinoma of the cervix. ${ }^{521}$ Indeed, one of these studies suggested that apoptosis could predict the prognosis in patients with carcinoma of the cervix treated by radiotherapy. ${ }^{5}$ These results are at variance with our own data, which do not suggest that apoptosis is of independent prognostic significance in carcinoma of the cervix. However, several other studies based on a variety of tumour types also show no correlation of apoptosis with prognosis or other known prognostic variables. ${ }^{22-25}$

While there is general acceptance that apoptosis is a fundamental mechanism in cell kinetics, it may be that the overall contribution of apoptotic cell death on its own to tumour kinetics is relatively small. This would mean that much larger studies than ours would be required to reveal any underlying statistically significant correlations with other prognostic variables and long term patient survival.

Some studies of apoptosis have been based on assessment of in situ labelling of DNA fragments. ${ }^{6727-29}$ While there is no doubt that these techniques may be useful in assisting the recognition of apoptosis, most investigators report a lack of specificity ${ }^{6229}$ as cells which are not apoptotic on morphological grounds may be labelled. ${ }^{4}{ }^{23}{ }^{24}{ }^{29}$ While this may reflect an ability to mark cells which are about to undergo apoptosis, ${ }^{23}{ }^{26}$ it is not certain whether this concept is valid. Studies using these methods tend to produce higher percentages of apoptosis $^{22}$ and this may influence the subsequent statistical analysis of the data. A more 
reliable marker for the process would not only make assessment of apoptosis more straightforward but it would also permit direct comparison between studies on different tumour types.

Review of the complexity of tumour cell kinetics suggests that studies incorporating other relevant factors, such as tumour cell proliferation and necrosis, may provide a more meaningful insight into tumour progression. In practical terms, however, it is difficult to see how the relative contributions of these factors to the overall kinetics of an individual tumour could be best assessed. There is a mechanistic link between apoptosis and tumour cell proliferation, ${ }^{30}$ and some studies have suggested a linear correlation between these two factors. ${ }^{19}$ Other work we have carried out, however, has failed to show a statistically significant relation between apoptosis and other measurements of tumour proliferation (unpublished). This may reflect a complex and unstable relation between apoptosis and cell proliferation .

The majority of studies of apoptosis have attempted to assess only viable tumour, but criteria for excluding necrotic tumour are variable, suggesting that in some cases necrotic cell death is at least partially included in the assessment of tumour cell loss. In this study we avoided areas of necrotic tumour, but in so doing we may have selected a subset of tumours which have a similar prognosis because of reduced tumour necrosis. Furthermore, excluding areas of necrosis may underestimate the contribution of apoptotic cell death to tumour progression and response to radiotherapy. Some studies have shown that a low oxygen microenvironment may result in selection of tumour cells with a reduced apoptotic potential, giving rise to more aggressive tumour progression. ${ }^{31}$ It has also been suggested that apoptotic cell death of endothelial cells contributing to the tumour's blood supply is a prime mechanism underlying tumour necrosis (Wyllie AH, personal communication).

In summary, we have shown that large scale light microscopic studies of apoptosis in tumours can be carried out in a semiautomated manner using the CAS 200 static image analysis system. The assessment of spontaneous levels of apoptosis in carcinoma of the cervix, however, provided no useful prognostic information in addition to that already derived from other more usual variables such as tumour stage. There seems little doubt that the relation between tumour cell proliferation and tumour cell loss, through apoptosis and necrosis, is complex and warrants further investigation as a means of predicting tumour progression.

1 Hickman JA. Apoptosis induced by anticancer drugs. Cancer Metast Rev 1992;11:121-39.

2 Kerr JFR, Searle J. Apoptosis: its native and kinetic role. In: Meyer RE, Withers HR, eds. Radiation biology in cancer research. New York: Raven Press, 1980:367-84.
3 Stephens LC, Ang KK, Schultheiss TE, et al. Apoptosis in irradiation mivine tumours. Radiat Res 1991;27:308-16.

4 Kerr JFR, Winterford CM, Harman BV. Apoptosis: its significance in cancer and cancer therapy. Cancer 1994;73: 2013-26.

5 Levine EL, Davidson SE, Roberts SA, et al. Apoptosis as a predictor of response to radiotherapy in cervical carcinoma. Lancet 1994;344:472.

6 Ansari B, Coates PJ, Greenstein BD, et al. In-situ end strand labelling detects DNA strand breaks in apoptosis and other physiological and pathological states. F Pathol 1993;170:18 .

7 Gavrieli Y, Sherman Y, Ben-Sasson SA. Identification of programmed cell death in situ via specific labelling of nuclear DNA fragmentation. F Cell Biol 1992;119:493501.

8 Harmsel BT, Smedt SF, Kuijpes J, et al. Bel-2 immunoreactivity increases with severity of CIN: a study of normal cervical epithelia, CIN and cervical carcinoma. F Pathol 1996; 179:26-30.

9 Swat W, Ignatowicz L, Kisuelow P. Detection of apoptosis in immature CD $4+8+$ thymocytes by flow cytometry. $\mathcal{F}$ Immunol Methods 1991;137:79-85.

10 Telford WS, King LE, Fraker PJ. Comparative evaluation of several DNA binding dyes in the detection of apoptosis associated chromatin degredation by flow cytometry. Cytometry 1992;13:137-42.

11 Hall PA, Coates PJ. Assessment of cell proliferation in pathology - what next? Histopathology 1995;16:105-12.

12 Kerr JFR, Wyllie AH, Currie AR. Apoptosis: a basic biological phenomenon with wide ranging implications in tissue kinetics. Br f Cancer 1972;26:239-57.

13 Wyllie AH, Kerr JFR, Currie AR. Cell death: the significance of apoptosis. Int Rev Cytol 1980;68:251-306.

14 Kernohan NM, Cox LS. Regulation of apoptosis by bcl-2 and its related proteins: immunocytochemical challenges and therapeutic implications. F Pathol 1996;179:1-3.

15 Lane DP. P53, guardian of the genome. Nature 1992;358: $15-16$.

16 Bolger BS, Symonds RP, Stanton PD, et al. Prediction of radiotherapy response of cervical carcinoma through measurement of proliferation rate. Br f Cancer 1996;74: 1223-6.

17 Sarraf CE, Bowen ID. Proportions of mitotic and apoptotic cells in a range of untreated experimental tumours. Cell Tissue Kinet 1988;21:45-9.

18 Leoncini L, Del Vecchio MT, Mehga T, et al. Correlation between apoptotic and proliferative indices in malignant non Hodgkin's lymphoma. Am f Pathol 1993;142:755-63.

19 Aihara M, Truong LD, Dunn JK, et al. Frequency of apoptotic bodies positively correlates with Gleason grade in prostatic cancer. Hum Pathol 1994;25:797-801.

20 Sasano H, Imatani A, Schizawa S, et al. Cell proliferation and apoptosis in normal and pathologic human adrenal. Mod Pathol 1995;8:11-17.

21 Sheets EE, Crum CP, Yeh J. Association between cervical neoplasia and apoptosis as determined by in situ nuclear labelling. Gynecol Oncol 1996;63:94-100

22 Todd D, Guang Y, Brown RW, et al. Apoptosis in renal cell carcinoma: detection by in situ end-labelling of fragmented DNA and correlation with other prognostic factors. Hum Pathol 1996;27:1012-17.

23 Shoji Y, Saegusa M, Takano Y, et al. Correlation of apoptosis with tumour cell differentiation, progression and HPV infection. F Clin Pathol 1996;49:134-8.

24 Shriohara T, Ohshina K, Murayama $\mathrm{H}$, et al. Apoptosis and proliferation in gastric carcinoma: the association with histological type. Histopathology 1996;29:123-9.

25 Lipponen PK, Haltomaa S. Apoptosis in bladder cancer as related to standard prognostic factors and prognosis. $\mathcal{F}$ Pathol 1994;173:333-9.

26 Hartley MK. Association between apoptotic index and established prognostic parameters in endometrial adenocarcinoma. Histopathology 1995;27:469-72.

27 Wijsman JH, Jonker RR, Keijzer R, et al. A new method to detect apoptosis in paraffin sections: in situ end labelling of fragmented DNA. F Histochem Cytochem 1993;41:7-12.

28 Gold R, Schmied M, Rothe G, et al. Detection of DNA fragmentation in apoptosis: application of in situ nick translation to cell culture systems and tissue systems and tissue sections. J Histochem Cytochem 1993;41:1023-30.

29 Gorczya W, Gong J, Darzynkiewicz Z, et al. Detection of DNA strand breaks in individual apoptotic cells by the in situ terminal deoxynucleotidyl transferase and nick translation assays. Cancer Res 1993;53:1945-51.

30 Evan GI, Wyllie AH, Gilbert CS, et al. Induction of apoptosis in fibroblasts by c-Myc protein. Cell 1992;69:119-28.

$31 \mathrm{Kim}$ CY, Tsai MH, Osmanian C, et al. Selection of human endocervical epithelial cells that possess reduced apoptotic potential to low oxygen conditions. Cancer Res 1997;57: 4200-4. 